\section{Experiencia de educación continua en línea en gastroenterología para médicos no especialistas}

\author{
LORENA ISBEJ ${ }^{1,2}$, JAVIER URIBE ${ }^{3}$, OLGA CARRASCO ${ }^{4}$, \\ ISAAC VILLARROEL ${ }^{5}$, MARGARITA PIZARRO ${ }^{6}$, \\ MARÍA ISABEL JIRÓN ${ }^{7}$, EDGAR SANHUEZA ${ }^{8}$, \\ MANUEL ÁLVAREZ-LOBOS ${ }^{6}$, CRISTIÁN HERNÁNDEZ-ROCHA ${ }^{6}$, \\ ANTONIO ROLLÁN ${ }^{8}$, XIMENA MONSALVE4,11, \\ LUIS ANTONIO DÍAZ ${ }^{12}$, MARÍA ALEJANDRA CERDA ${ }^{4,13}$, \\ TOMÁS KRAMER ${ }^{4,14}$, FERNANDO MUNIZAGA ${ }^{4,15}$, \\ ARNOLDO RIQUELME 3 3,4,6,10
}

\section{Experience of continuing online education in gastroenterology for non specialist medical doctors}

Background: Continuing education is essential for health professions and online courses can be a good way for professional development. Aim: To describe the experience with online courses for continuing education in hepatology and gastroenterology and to analyze their educational impact. Material and Methods: A three years' experience in courses on liver diseases and digestive tract is described. Their curricular design, methodology, and the educational impact was analyzed using the four levels of the Kirkpatrick's model. Results: On average, there were 321 students per course (2015-2017). 94\% were Chilean and 6\% from abroad (20 countries). In the educational impact analysis, in level 1 "reaction": 93\% said that the course fulfilled their expectations and $92 \%$ would recommend it. In level 2 "learning": $42 \%$ approved the courses. Level 3 "behavior" was not evaluated and level 4 "organizational change" highlighted that the traditional face-to-face continuing education model of Chilean Gastroenterology Society (SChG) changed to full distance model in these three courses, with 1284 students from South America, Asia and Europe, in a 3-years-period. Additionally, these programs were included in the Medical Society of Santiago (SMS) continuing education agenda. Conclusions: The alliance between the SMS and the SCh G generated on line courses that meet the educational needs of physicians and medical students, with excellent results and student perception.

(Rev Med Chile 2019; 147: 1059-1066)

Key words: Education, Distance; Education, Medical, Continuing; Gastroenterology; Program Evaluation.
'Escuela de Odontología, Facultad de Medicina, Pontificia Universidad Católica de Chile. Santiago, Chile.

${ }^{2}$ Unidad Docente de Farmacología y

Toxicología, Facultad de Medicina, Pontificia Universidad Católica de Chile. Santiago, Chile.

${ }^{3}$ Escuela de Medicina, Facultad de Medicina, Pontificia Universidad Católica de Chile. Santiago, Chile.

${ }^{4}$ Comité de Educación Médica Continua, Sociedad Médica de Santiago. Santiago, Chile.

${ }^{5}$ Compuerta Estudio Virtual. Santiago, Chile. ${ }^{6}$ Departamento de Gastroenterología,

Escuela de Medicina, Facultad de Medicina, Pontificia Universidad Católica de Chile.

Santiago, Chile.

${ }^{7}$ Departamento de Gastroenterología, Hospital del Salvador, Sede Oriente, Facultad de Medicina, Universidad de Chile. Santiago, Chile.

${ }^{8}$ Unidad de Gastroenterología. Facultad de Medicina Clinica Alemana-Universidad del Desarrollo. Santiago, Chile.

9Departamento de Ciencias de la Salud y Centro de Educación Médica, Facultad de Medicina, Pontificia Universidad Católica de Chile. Santiago, Chile.

${ }^{10}$ Sociedad Chilena de Gastroenterología. Santiago, Chile.

${ }^{11}$ UDA Hospital Dr. Sótero del Río, Facultad de Medicina, Pontificia Universidad Católica de Chile. Santiago, Chile.

${ }^{12}$ Departamento de Medicina Interna, Escuela de Medicina, Facultad de Medicina, Pontificia Universidad Católica de Chile. Santiago, Chile.

${ }^{13}$ Facultad de Medicina, sede norte, Hospital Clínico José Joaquín Aguirre. Universidad de Chile. Santiago, Chile.

${ }^{14}$ Residente de programa de Medicina Interna. Facultad de Medicina. Universidad de los Andes. Santiago, Chile. ${ }^{15}$ Departamento de Medicina Interna, Campus Centro, Hospital San Borja Arriarán. Facultad de Medicina, Universidad de Chile. Santiago, Chile.

Trabajo no recibió financiamiento. Los autores declaran no tener conflictos de interés.

Recibido el 30 de enero de 2019, aceptado el 5 de agosto de 2019.

Correspondencia a:

Dra. Lorena Isbej Espósito

Profesor Asistente. Escuela de Odontología Facultad de Medicina. Pontificia Universidad Católica de Chile. Av. Vicuña Mackenna 4860, Macul. Santiago, Chile.

lisbeje@uc.cl 
L os médicos generales deben alcanzar diferentes competencias o metas de aprendizaje como resultado de su proceso educativo, es decir, un conjunto de habilidades observables adquiridas exitosamente, y que pueden ser medidas como resultado de la combinación e integración de conocimientos, destrezas y actitudes ${ }^{1,2}$. Para guiar este proceso, en nuestro país existen perfiles de egreso de las escuelas de medicina adscritas a la asociación de facultades de medicina de Chile (ASOFAMECh), que determinan las competencias que los médicos generales deben demostrar al ser evaluados en el Examen Único Nacional de Conocimientos de Medicina (EUNACOM) ${ }^{3-7}$. Sin embargo, aún existe variabilidad en los perfiles de egreso obtenidos en las diferentes instituciones ${ }^{3}$.

Luego que el médico egresa, el avance constante del conocimiento y la necesidad de tomar decisiones clínicas basadas en la evidencia, impone el desafío de la actualización permanente como parte del compromiso social y profesionalismo ${ }^{8}$. Para ello, existe la educación médica continua, cuyos objetivos fueron vislumbrados por Jehan Saleh en la universidad de Teherán hace más de cincuenta años ${ }^{9}$ y que son constantemente revisados y actualizados por instituciones como la World Federation for Medical Education ${ }^{8}$. Se define educación médica continua como "cualquier actividad que tenga como objetivo mantener, desarrollar o aumentar el conocimiento, las habilidades y las relaciones profesionales que utiliza un médico para proporcionar servicios a los pacientes, el público o la profesión"10,11.

Estos antecedentes, tanto los perfiles de egreso heterogéneos como la necesidad de formación médica continua, hacen que la actualización a través de diferentes tipos de cursos sea fundamental por el impacto que tendría en la atención de salud y para lo cual la educación a distancia en su modalidad "on line" surge como una alternativa innovadora y útil, no sólo para el desarrollo profesional continuo de los egresados de escuelas de medicina de Chile, sino también para médicos no especialistas formados fuera del país ${ }^{12-15}$.

Se suma a lo anterior los cambios en educación médica, donde una de las principales transformaciones es la incorporación del concepto de "evaluación para el aprendizaje" (EPA), el cual propone una renovación en la forma de entender la evaluación, ayudando a los estudiantes a reconocer lo que se espera de ellos y a la vez hacerlos parte de su formación. Además, esta EPA-permite la entrega de retroalimentación individualizada, lo que logra mayor motivación por parte de los alumnos en el proceso de aprendizaje $\mathrm{e}^{16-18}$.

Frente a los escenarios mencionados y a la continua necesidad de perfeccionamiento en la profesión médica, es que en el año 2015 la Sociedad Chilena de Gastroenterología (SCHGE) y la Sociedad Médica de Santiago (SMS)-Sociedad Chilena de Medicina Interna, deciden iniciar un innovador proyecto. Este consiste en ofrecer un programa de educación continua completamente a distancia u "online" para estudiantes de pregrado y médicos no especialistas, que incluye tres cursos para patologías específicas Enfermedades Hepáticas (C-EH), Tubo Digestivo Alto (C-TDA) y Tubo Digestivo Bajo (C-TDB), favoreciendo así la actualización de diagnóstico y tratamiento de las enfermedades más frecuentes en la práctica clínica en gastroenterología y hepatología.

La elección de contendidos de gastroenterología, como tópicos a abordar en estos cursos, se sustenta en la importancia de la patología de tubo digestivo, siendo el cáncer gástrico y de vesícula las neoplasias con mayor mortalidad en la población chilena. Por otra parte, la cirrosis hepática, con una tasa de mortalidad 16,6/100.000 habitantes, es una de las causas específicas de mortalidad más importantes en nuestro país ${ }^{19}$. Esto, sumado a la alta frecuencia de consulta por patología gastroenterológica en los servicios de urgencia, en donde sólo la diarrea aguda constituye entre 4 a 4,3\% de las consultas en los servicios de urgencia públicos del país ${ }^{20}$, son razones más que suficientes para la realización de cursos sobre el manejo de este grupo de patologías de alta prevalencia en Chile e Iberoamérica.

Los objetivos de esta publicación son describir la experiencia en educación continua de cursos on line de hepatología y gastroenterología en Chile e Iberoamérica, y analizar el impacto educacional logrado a través del modelo de Kirkpatrick.

\section{Métodos}

El proceso de matrícula de los C-EH, C-TDA y C-TDB se realizó de manera completamente on line, lo que facilitó la inscripción para alumnos nacionales y extranjeros. Se estableció un arancel de referencia a pagar al cual se le aplicaron descuentos según la calidad del postulante. 
Para el diseño curricular se utilizó el modelo de $K e r n^{21}$, donde el primer paso es la identificación y análisis crítico de necesidades de atención médica u otro problema, luego se evalúan las necesidades específicas (las del grupo al que va dirigido). El tercer paso tiene relación con el propósito y objetivos de aprendizaje del programa, reconociendo las necesidades de los estudiantes. El paso cuatro hace referencia a las estrategias educativas, donde se escogen los métodos para lograr de mejor manera los objetivos planteados. El quinto paso es la implementación, tanto de la intervención educativa como de su evaluación, lo que es crítico para el éxito, ya que transforma un ejercicio teórico en una realidad. Finalmente, el sexto paso corresponde a la evaluación y feedback de los estudiantes y del plan de estudios en general, el que puede ser formativo o sumativo. Esta forma de diseño curricular les entrega a los cursos un fuerte sustento teórico con base en la literatura sobre educación médica disponible ${ }^{22}$.

Las metodologías de enseñanza y aprendizaje fueron completamente a distancia, de manera asincrónica ${ }^{23,24}$, y los cursos incluyeron las patologías más frecuentes y de mayor relevancia en la población chilena, que se agruparon en módulos que consideraron un número similar de contenidos. Se contó con clases video grabadas por expertos iberoamericanos con tecnología Full HD, con un alto nivel de iluminación, audio, y animaciones, en caso que fuese necesario. Luego del proceso de grabación, las presentaciones fueron revisadas en conjunto con el profesor a cargo, para cerciorarse del cumplimiento de los objetivos planteados.

La evaluación de los cursos se estructuró utilizando el modelo de EPA ${ }^{18}$, donde las preguntas de opción múltiple fueron construidas con viñetas clínicas, siguiendo los criterios de Haladyna ${ }^{25,26}$.

Todas las evaluaciones fueron de carácter obligatorio, incluyendo una prueba diagnóstica (formativa), dos evaluaciones sumativas y una prueba final, que incluyó todas las clases del curso. Para todas las pruebas, el grupo de alumnos fue dividido en dos según sus apellidos y se les asignó horarios específicos para rendir la evaluación. La nota mínima de aprobación del curso fue 5,0 debiendo además rendir la totalidad de las evaluaciones.

Al final de cada módulo se realizó una retroalimentación video grabada de manera asincrónica.

Para medir el impacto de los cursos, se utilizó el modelo de Kirkpatrick, que propone medir la efectividad de una intervención educacional clasificándola en 4 niveles: el primero corresponde a reacción, el segundo a aprendizaje, el tercero a conducta o transferencia de lo aprendido y el cuarto a impacto en la institución ${ }^{27,28}$.

Los alumnos respondieron encuestas de percepción, evaluando utilidad del tema, calidad de presentaciones, cumplimiento de expectativas, sugerencias sobre las pruebas, si recomienda el curso e interés de seguir participando en futuros cursos.

Este trabajo cuenta con la aprobación de la Mesa Directiva de la SMS y del Directorio de la SCHGE.

\section{Resultados}

Se diseñaron 3 tipos de cursos, que tenían entre 17 y 20 clases, todas on line, separadas en 4 o 5 módulos, más un tópico ético, con una duración de 3 meses cada uno.

Entre los años 2015 a 2017 se realizaron 4 cursos (1 C-TDA, 1 C-TDB y 2 C-EH) con un total de 1.284 alumnos (promedio 321 alumnos por curso), 56\% hombres y $44 \%$ mujeres. El desglose de los matriculados se muestran en las Figuras 1.1 y 1.2 .

Si bien, las matrículas fueron principalmente de nacionalidad chilena, también hubo alumnos de gran parte de Sudamérica, Asia y Europa (Figura 2). Casi la mitad de los alumnos chilenos era de Santiago, y el resto presentó una distribución variada, desde el extremo norte del país hasta la zona más austral (Figura 3).

Cincuenta y nueve por ciento de los matriculados fueron médicos, seguidos de estudiantes y residentes-becados que representaron $38 \%$ y $5 \%$ de los inscritos, respectivamente.

Entre los médicos, existió una amplia distribución del año de titulación, desde 1973 hasta el 2016, siendo este último año, junto al 2014 y 2015, los que representaron al mayor número de matriculados, ( $45 \%$ de todos los inscritos).

Utilizando el modelo Kirkpatrick, el primer nivel o de "reacción" se cumplió de manera satisfactoria. A través de la encuesta de percepción, los alumnos evaluaron de manera favorable el curso, calificándolo en su mayoría con nota entre 6 y 7 , evidenciándose de manera cualitativa siempre "muy satisfechos con el curso". Noventa y tres por 


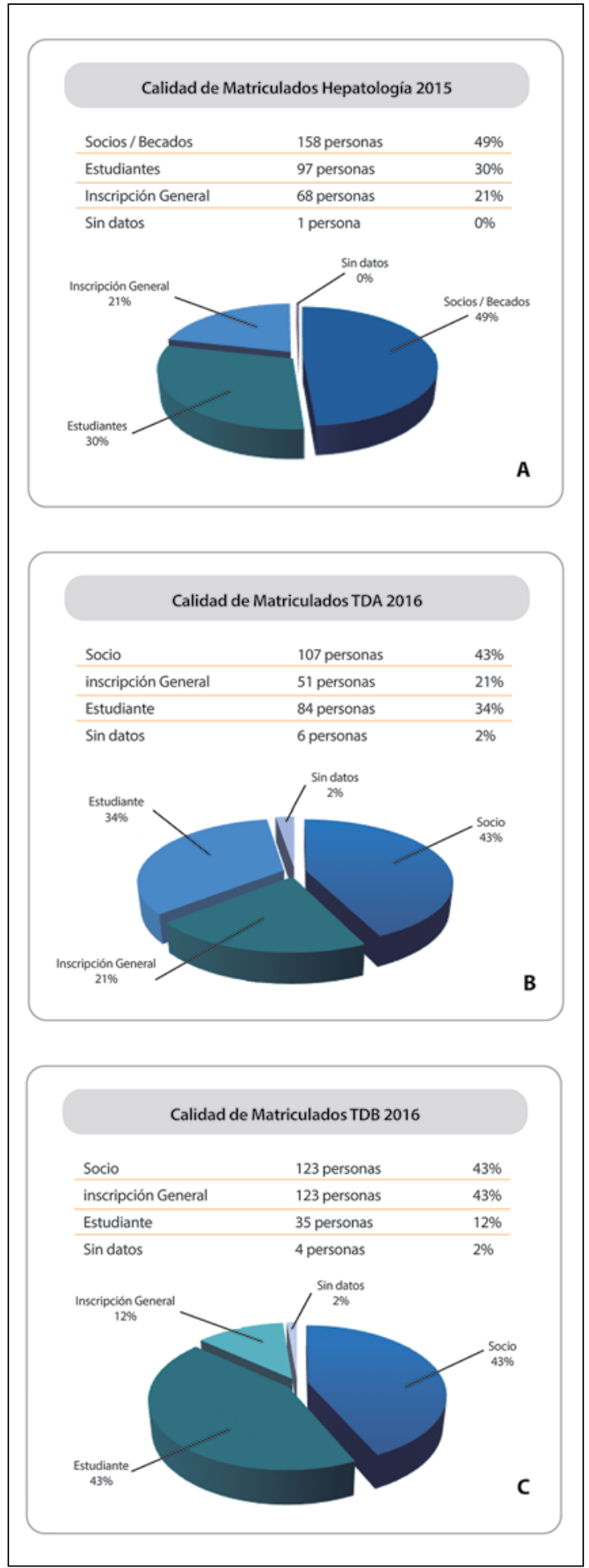

Figura 1.1. Calidad de matriculados en cursos a distancia. A: Curso Hepatología (2015); B: Curso Tubo Digestivo Alto (2016); C: Curso Tubo Digestivo Bajo (2016).

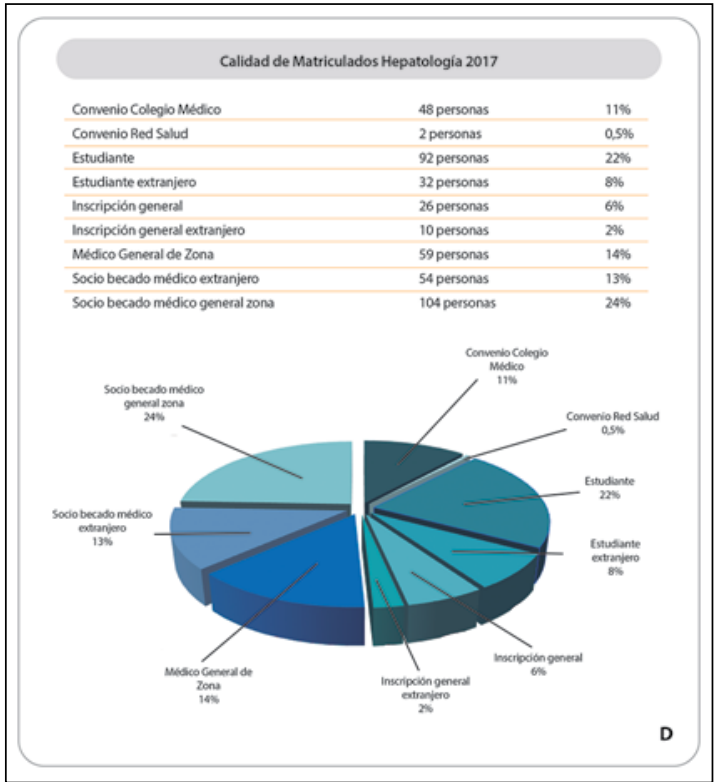

Figura 1.2. Calidad de matriculados en cursos a distancia. D: Curso Hepatología (2017).

ciento señaló que el curso cumplió sus expectativas, $92 \%$ lo recomendaría y $86 \%$ se mostró interesado en participar en nuevas versiones de éste.

En relación a las exposiciones, destaca que $97 \%$ de los alumnos las calificó como adecuadas, mientras que ninguno utilizó el adjetivo inadecuada. El material audio visual y los recursos de apuntes de referencia fueron también evaluados de manera positiva, obteniendo sobre $95 \%$ de aceptación.

Finalmente, al observar la evaluación de la utilidad de los temas seleccionados, 58\% la califica con nota máxima $(7,0)$ y $62 \%$ le otorga la misma nota de manera global al curso.

El segundo nivel, "aprendizaje", se evidencia en una buena tasa de aprobación y rendimiento en las pruebas aplicadas. Del total de alumnos inscritos en los cursos, $42 \%$ aprobó los cursos. Las evaluaciones fueron efectuadas sin complicaciones en $89 \%$ de los casos, pero destaca el hecho de que $18 \%$ de los participantes señaló que el tiempo asignado para ellas no era el adecuado.

La participación en las evaluaciones fue decreciendo, pasando de $72 \%$ de participación en la primera prueba, a $57 \%$ en la evaluación final. La evaluación de los participantes se detalla en la Tabla 1. 


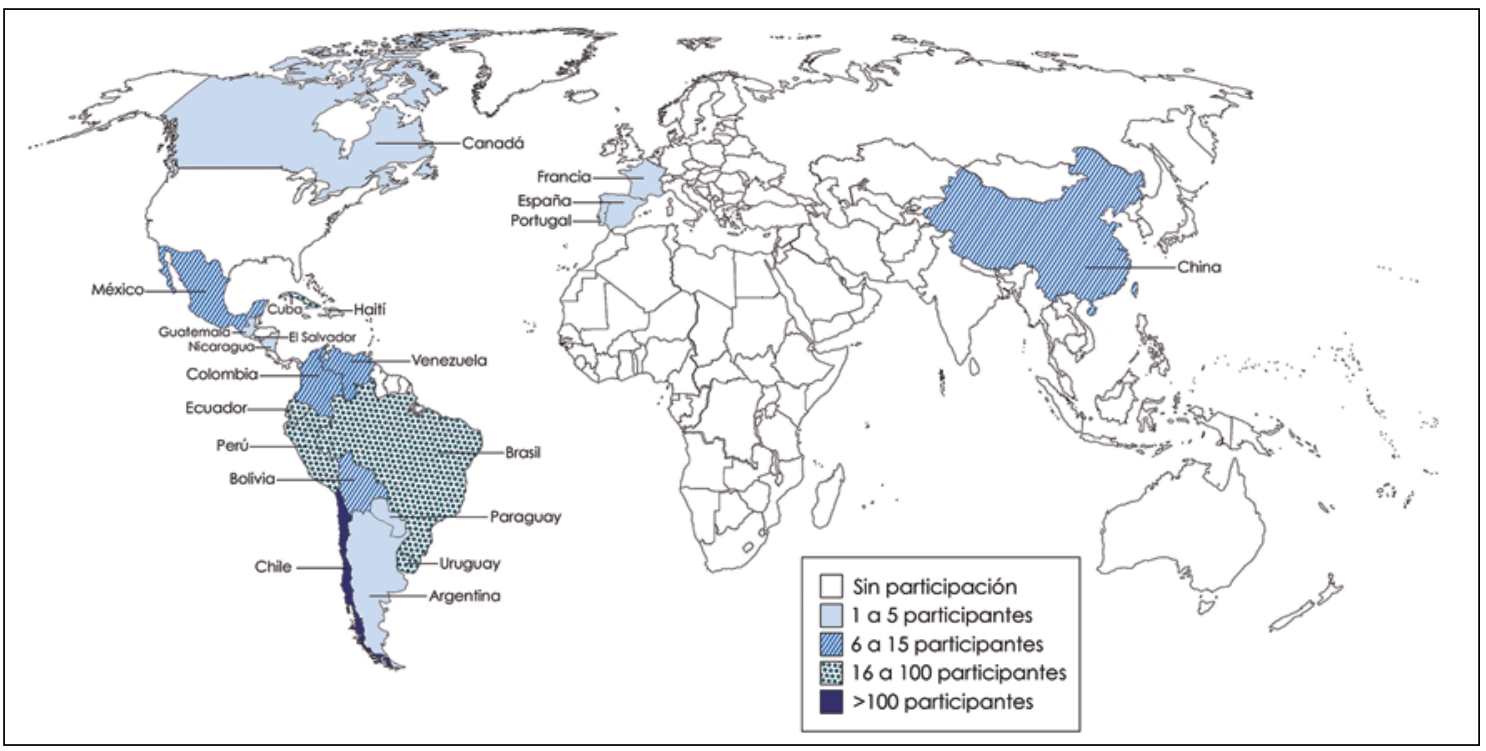

Figura 2. Nacionalidad de participantes matriculados en los cursos.

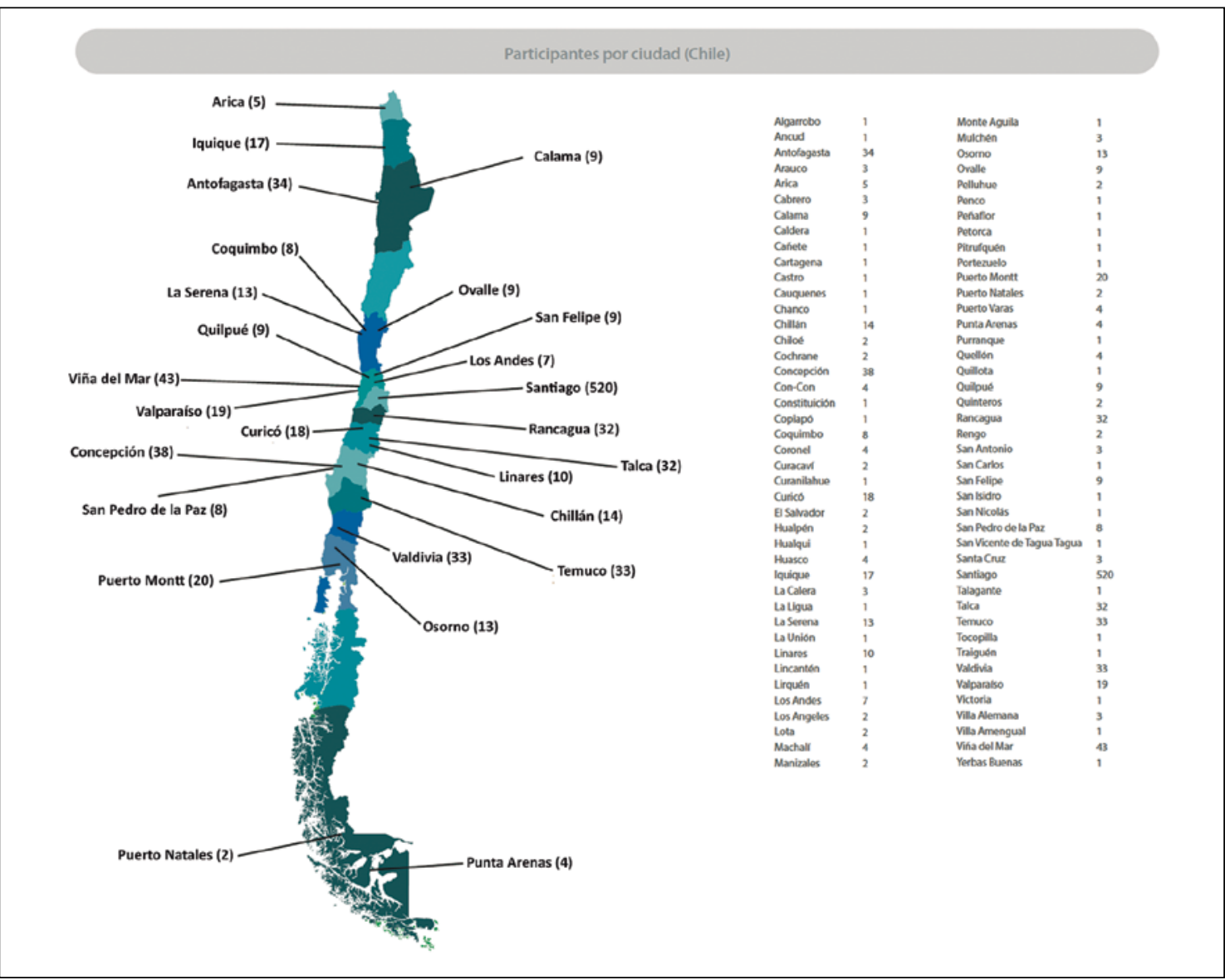

Figura 3. Participantes de nacionalidad chilena por ciudad. 
Tabla 1. Resultado evaluación participantes. Participación de alumnos en el proceso evaluativo cursos Hepatología 2015, TDA 2016, TDB 2016 y Hepatología 2017

\begin{tabular}{|lcc|}
\hline & $\begin{array}{c}\text { Total de } \\
\text { alumnos }\end{array}$ & $\begin{array}{c}\text { \% del total } \\
\text { de alumnos }\end{array}$ \\
\hline Prueba 1 & 1.026 & 80 \\
\hline Prueba 2 & 889 & 69 \\
\hline Prueba final & 818 & 64 \\
\hline Alumnos aprobados & 674 & 52 \\
\hline Alumnos reprobados & 610 & 48 \\
\hline
\end{tabular}

El nivel 3, "conducta", no fue evaluado formalmente, aunque muchos alumnos reportaron que los contenidos "les servirán para ser aplicados en sus trabajos".

Finalmente, el nivel 4, "cambios organizacionales o institucionales", sí ha logrado el impacto originalmente proyectado por el comité de educación de la SChGE y la SMS, ya que los cursos se han realizado de manera sistemática y son parte de la agenda de educación continua de la SMS. Este cambio implica una transformación desde la mirada tradicional de la educación continua presencial (curso de avance y congreso anual de la SChGE) a una apertura a la educación a distancia con proyección nacional e internacional (Figura 2).

\section{Discusión}

Actualmente, la educación continua y el perfeccionamiento constante son pilares fundamentales en la formación de los profesionales de la salud. En este contexto, el uso de plataformas online ha demostrado ser capaz de llevar contenidos actualizados a médicos chilenos e hispanoparlantes, constituyendo sin duda un instrumento efectivo para la democratización del conocimiento, lo que podría tener un impacto en el mejor manejo de los pacientes con diferentes patologías.

Una de las fortalezas más importantes de estos cursos de educación continua en gastroenterología, es el diseño de su currículo en base al modelo de 6 pasos de Kern, que tiene como finalidad "proporcionar un enfoque práctico y teóricamente sólido para desarrollar, implementar, evaluar y mejorar continuamente las experiencias educativas en medicina" 21 .
Por otro lado, tanto las clases y los expositores, así como también el material audiovisual, recibieron una positiva evaluación por parte de los alumnos, que se relaciona con la selección de oradores con liderazgo a nivel nacional e iberoamericano, sumado al uso de los capítulos del libro de diagnóstico y tratamiento de las enfermedades digestivas de la SChGE, que se entrega en versión digital a cada participante de los cursos, constituyéndose como un importante material instruccional que puede ser actualizado y utilizado como base para la realización de futuras versiones ${ }^{29}$.

Si bien, $42 \%$ de los alumnos logró aprobar, debemos considerar que los cursos de la SMS tienen un punto de corte de aprobación más alto (nota 5,0 en escala $1,0-7,0$ ) y que supera $60 \%$ del puntaje habitualmente utilizado como método holístico para nota 4,0. Este porcentaje de aprobación es superior a los evidenciados en cursos masivos en línea como los MOOC's (Massive Open Online Courses), que suelen ser inferiores a $20 \%{ }^{30,31}$.

Entre los aspectos por mejorar, destaca el hecho de que existe un gran porcentaje de estudiantes que no rinde la totalidad de las pruebas. Esta situación puede relacionarse con dificultades en el acceso a la plataforma on line en la evaluación final del curso, y una ventana de tiempo acotada para la realización de las pruebas. Estos puntos deben ser considerados en las próximas versiones del curso, para así poder mejorar aún más la percepción y rendimiento de éste.

Pese a la gran cantidad de contenidos abordados por los cursos, existen algunos que quedan sin abarcar y que otorgan la oportunidad de incluirlos en nuevas versiones de éstos o generar nuevos programas que, con una modalidad de enseñanza similar, se encarguen de dar respuesta a esta necesidad expresada al finalizar estos módulos.

\section{Conclusiones}

Cabe destacar la alianza generada entre la Sociedad Médica de Santiago, con 149 años de experiencia en actividades docentes, la Sociedad Chilena de Gastroenterología y Asociación Latinoamericana para el Estudio del Hígado que, pese a su juventud y reciente formación, ha sido capaz de generar hasta el momento 4 cursos a distancia que dan respuesta a necesidades educativas de médicos y estudiantes de medicina, con una sólida 
base teórica en educación médica y con excelentes resultados y percepción de sus alumnos. Es de esperar que esta productiva alianza se mantenga a futuro, incluyendo una reciente asociación con la Organización Panamericana de Gastroenterología, para potenciar de manera colaborativa, la realización de cursos de calidad, en subdisciplinas de la gastroenterología.

Esperamos que estas experiencias sirvan como modelo para poder generar nuevos cursos que abarquen contenidos de las distintas especialidades médicas y así dar respuesta a una creciente necesidad de perfeccionamiento existente en nuestro país y en países hispanoparlantes.

\section{Referencias}

1. Callahan D, Crosby J, Davies D, Davis M, Dollase R, Friedman M, et al. AMEE Medical Education Guide No 14: Outcome-based education. Med Teach 2014; 44 (14).

2. Frank JR, Snell LS, Cate O Ten, Holmboe ES, Carraccio C, Swing SR, et al. Competency-based medical education: Theory to practice. Med Teach 2010; 32 (8): 63845.

3. Parada M, Romero MI, Moraga F. Perfiles de egreso de las carreras de medicina en Chile. Rev Med Chile 2015; 143 (4): 512-9.

4. Rosso P, Velasco NMR. Undergraduate curriculum reform at the Pontifical Catholic University Medical School: aims, methodology and advance status. Rev Med Chile 1997; 125 (7): 796-807.

5. Sánchez I, Riquelme A, Moreno R, Mena B, Dagnino J. Revitalising medical education: the School of Medicine at the Pontificia Universidad Católica de Chile. Clin Teach 2008; 5: 57-61.

6. Herskovic LP. La Reforma Curricular de la Escuela de Medicina de la Universidad de Chile. Rev Chil Pediatr 2015; 16 (1): 9-11.

7. Cisternas M, Rivera S, Sirhan M, Thone N, Valdés C, Pertuzé J, et al. Reforma curricular de la carrera de Medicina de la Pontificia Universidad Católica de Chile. Rev Med Chile 2016; 144 (1): 102-7.

8. WFME Office. Continuing Professional Development of Medical Doctors WFME Global Standards. Denmark; 2015.

9. Saleh J. The objectives and problems of continuing medical education. In: Medicine a lifelong study. Proceedings of the 2nd World Conference on Medical Education. In: 1959. Chicago: World Medical Association;
10. Davis N, Davis D, Bloch R. Continuing medical education: AMEE Education Guide No 35. Med Teach. 2008; 30 (7): 652-66.

11. Van Nieuwenborg L., Goossens M., De Lepeleire J. et al. Continuing medical education for general practitioners: A practice format. Postgrad Med J 2016; 92 (1086): 21722.

12. Harden RM. A new vision for distance learning and continuing medical education. J Contin Educ Health Prof 2005; 25 (1): 43-51.

13. Bernard RM, Abrami PC, Lou Y, Borokhovski E, Wade A, Wozney L, et al. How Does Distance Education Compare With Classroom Instruction? A Meta-Analysis of the Empirical Literature. Rev Educ Res 2004; 74 (3): 379-439.

14. Ellaway R, Masters K. AMEE Guide 32: E-Learning in medical education Part 1: Learning, teaching and assessment. Med Teach 2008; 30 (5): 455-73.

15. MacWalter G, McKay J, Bowie P. Utilisation of internet resources for continuing professional development: a cross-sectional survey of general practitioners in Scotland. BMC Med Educ 2016; 16 (1): 24.

16. Shumway JM, Harden RM. AMEE guide no. 25: The assessment of learning outcomes for the competent and reflective physician. Med Teach 2003; 25 (6): 569 84.

17. Krupat Jules L. ED. Commentary: Assessment Is an Educational Tool. Acad Med 2009; 84 (5): 548-50.

18. Schuwirth LWT, Van der Vleuten CPM. Programmatic assessment: from assessment of learning to assessment for learning. Med Teach 2011; 33 (6): 478-85.

19. Alonso F, Garmendia ML, De Aguirre M, Searle J. Análisis de la tendencia de la mortalidad por cirrosis hepática en Chile: Años 1990 a 2007. Rev Med Chile 2010; 138 (10): 1253-8. Available from: http:// www.scielo.cl/scielo.php?script $=$ sci_arttext\&pid= S0034-98872010001100007\&lng=en\&nrm=iso\&tlng=en [citado el 9 de octubre de 2018].

20. Gobierno de Chile MdS. Departamento de Estadísticas e Información de Salud [Internet]. Available from: http:// www.deis.cl/estadisticas-atencionesurgencia.

21. Thomas PA, Kern DE, Hughes MT CB. Curriculum development for medical education:a six-step approach. Johns Hopkins University Press, editor. 2015.

22. Langendyk V, Mason G, Wang S. How do medical educators design a curriculum that facilitates student learning about professionalism? Int J Med Educ 2016; 7: 32-43.

23. Vaona A, Banzi R, Kh K, Rigon G, Cereda D, Pecoraro $\mathrm{V}$, et al. E-learning for health professionals (Review). Cochrane Database Syst Rev 2018; (1). 
24. Taylor DCM, Hamdy H. Adult learning theories : Implications for learning and teaching in medical education : AMEE Guide No . 83. Med Teach 2013; 1-12.

25. Haladyna TM, Downing SM. A Taxonomy of Multiple-Choice Item-Writing Rules. Appl Meas Educ 1989; 2 (1): 37-50.

26. Kattan E, Pérez G, Le Roy C, Sirhan M, González A, Rybertt T, et al. Proceso de mejoría de pruebas de conocimiento con preguntas de selección múltiple en un curso teórico de pregrado de medicina. Rev Educ Cienc Salud 2014; 11 (2): 116-23.

27. Yardley S, Dornan T. Kirkpatrick's levels and education “evidence". Med Educ 2012; 46 (1): 97-106.

28. Kirkpatrick DL, Kirkpatrick JD. Evaluating Training Programs. 3rd Editio. San Francisco: Berrett-Koehler
Publishers, Inc.; 2006. 380 p.

29. Weitz JC, Berger Z, Sabah S SH. Diagnóstico y tratamiento de enfermedades digestivas [Internet]. Santiago: Sociedad Chilena de Gastroenterología; 2013. Available from: http://www.sociedadgastro.cl/gastroweb/documentos/Diagnostico-y-tratamiento-ED. pdf $\#$ page $=219$.

30. Jordan K. Initial trends in enrolment and completion of massive open online courses Massive Open Online Courses. Int Rev Res Open Distance Learn 2014; 15 (1): 133-60.

31. Henderikx MA, Kreijns K, Kalz M. Refining success and dropout in massive open online courses based on the intention-behavior gap. Distance Educ 2017; 38 (3): 353-68. 